Research Article

\title{
A Variational Method for Multivalued Boundary Value Problems
}

\section{Droh Arsène Béhi $(\mathbb{D})$ and Assohoun Adjé}

UFR Mathématiques et Informatique, Université Félix Houphouet Boigny, Cocody, Abidjan 22 BP 582, Côte d'Ivoire

Correspondence should be addressed to Droh Arsène Béhi; arsene.behi@gmail.com

Received 18 September 2019; Revised 18 December 2019; Accepted 27 December 2019; Published 21 January 2020

Academic Editor: Simeon Reich

Copyright (C) 2020 Droh Arsène Béhi and Assohoun Adjé. This is an open access article distributed under the Creative Commons Attribution License, which permits unrestricted use, distribution, and reproduction in any medium, provided the original work is properly cited.

In this paper, we investigate the existence of solution for differential systems involving a $\phi$-Laplacian operator which incorporates as a special case the well-known $p$-Laplacian operator. In this purpose, we use a variational method which relies on Szulkin's critical point theory. We obtain the existence of solution when the corresponding Euler-Lagrange functional is coercive.

\section{Introduction}

This paper is devoted to the study of the following secondorder differential systems:

$$
\left\{\begin{array}{c}
\left.-\left(\phi\left(u^{\prime}(t)\right)\right)^{\prime}+\varepsilon \phi(u(t))=\nabla F(t, u(t)) \text { a.e.on } \Omega=\right] 0, T[ \\
\left(\phi\left(u^{\prime}(0)\right),-\phi\left(u^{\prime}(T)\right)\right) \in \partial j(u(0), u(T))
\end{array}\right.
$$

where $\varepsilon \geq 0$ is fixed, $\phi: \mathbb{R}^{N} \longrightarrow \mathbb{R}^{N}$ is a monotone homeomorphism, $j: \mathbb{R}^{N} \times \mathbb{R}^{N} \longrightarrow(-\infty,+\infty]$ is a proper, convex, and lower semicontinuous function, $F: \Omega \times \mathbb{R}^{N} \longrightarrow \mathbb{R}^{N}$ is a Caratheodory mapping, continuously differentiable with respect to the second variable and satisfies some usual growth conditions, $\nabla F(t, x)$ is the gradient of $F(t,$.$) at x \in \mathbb{R}^{N}$ and $\partial j$ denotes the subdifferential of $j$ in the sense of convex analysis.

Second-order differential problems with multivalued boundary value conditions have been studied by many authors. In this direction, we can cite the works of Bader and Papageorgioua [1], Béhi et al. [2], Gasinski and Papageorgiou [4], Jebelean [5], Jebelean and Morosanu [6], and references therein. In $[1,4-6]$, the authors investigate differential systems driven by a homogeneous
p-Laplacian operator while in problem (1), we deal with a nonhomogeneous $\phi$-Laplacian operator which incorporates as a special case the $p$-Laplacian operator. As a consequence, problem (1) is a generalization of the following problem studied, in 2005, by Jebelean and Morosanu [6]:

$$
\left\{\begin{array}{c}
\left.-\left(\phi_{p}\left(u^{\prime}(t)\right)\right)^{\prime}+\varepsilon \phi_{p}(u(t))=\nabla F(t, u(t)) \text { a.e. on } \Omega=\right] 0, T[ \\
\left(\phi_{p}\left(u^{\prime}(0)\right),-\phi_{p}\left(u^{\prime}(T)\right)\right) \in \partial j(u(0), u(T))
\end{array}\right.
$$

where $\phi_{p}$ denotes the homogeneous operator $p$-Laplacian. Indeed, in our work, the $\phi$-Laplacian operators are nonhomogeneous and are of the form $\phi(x)=a(x) \phi_{p}(x)$ with $\phi_{p}$ the $p$-Laplacian operator, $p>1$, and $a: \mathbb{R}^{N} \longrightarrow \mathbb{R}_{+}^{*}$ a continuous map.

In order to obtain existence result for problem (1), we will use a variational method which relies on Szulkin's critical point theory [7].

This paper is organized as follows: After introducing notations and preliminary results in Section 2, in Section 3 we give a variational approach to problem (1). In Section 4, using some results from Section 3, we prove our main result. In 
Section 4, we give an example to illustrate the applicability of our result. Finally, Section 5 is reserved for the conclusion.

\section{Preliminaries}

Let us recall some notions and results in the framework of Szulkin's critical point theory [7] which are needed and also some notations which will be used in the sequel. At this end, $W^{1, p}\left((0, T), \mathbb{R}^{N}\right)$ is the Sobolev Banach space which will be endowed with the norm:

$$
\|u\|_{m}=\left(m\|u\|_{p}^{p}+\left\|u^{\prime}\right\|_{p}^{p}\right)^{1 / p}
$$

where $m>0$ and $\|.\|_{p}$ is the norm on $L^{p}\left((0, T), \mathbb{R}^{N}\right)$ defined by

$$
\|u\|_{p}=\left(\int_{0}^{T}|u|^{p} \mathrm{~d} t\right)^{1 / p}
$$

We denote $\|\cdot\|_{\infty}$, the norm on the set $C\left([0, T], \mathbb{R}^{N}\right)$ :

$$
\|u\|_{\infty}=\max \{|u(t)|: t \in[0, T]\} .
$$

Let $(X,\|\|)$ be a real Banach space and $B: X \longrightarrow$ $(-\infty,+\infty]$ be a functional of type

$$
B=E+G \text {, }
$$

where $E \in C^{1}(X, \mathbb{R})$ and $G$ is proper, convex, and lower semicontinuous (lsc). A point $u \in X$ is said to be a critical point of $B$ if it satisfies the inequality

$$
\left\langle E^{\prime}(u), v-u\right\rangle+G(v)-G(u) \geq 0, \quad \forall v \in X .
$$

A number $c \in \mathbb{R}$ such that $B^{-1}(c)$ contains a critical point is called a critical value of $B$.

To establish existence results for (1), we will need the following proposition due to Szulkin (see [7] Proposition 1.1).

Proposition 1. If B satisfies (7), each local minimum point of $B$ is necessarily a critical point of $B$.

The functional $B$ is said to satisfy the Palais-smale (in short, (PS)) condition if every sequence $\left\{u_{n}\right\} \subset X$ for which $B\left(u_{n}\right) \longrightarrow c \in \mathbb{R}$ and

$$
\left\langle E^{\prime}\left(u_{n}\right), v-u_{n}\right\rangle+G(v)-G\left(u_{n}\right) \geq \varepsilon_{n}\left\|v-u_{n}\right\|, \quad \forall v \in X,
$$

where $\varepsilon_{n} \longrightarrow 0$ possesses a convergent subsequence.

\section{A Variational Approach for Problem (1)}

Before beginning the variational approach, we make the following hypotheses on the data of problem (1):

$\left(H_{a}\right): a: \mathbb{R}^{N} \longrightarrow \mathbb{R}_{+}^{*}$ is a continuous map such that there exists $\eta, M>0$ such that

$$
\eta \leq a(x) \leq M, \quad \forall x \in \mathbb{R}^{N} .
$$

$\left(H_{\phi}\right): \phi: \mathbb{R}^{N} \longrightarrow \mathbb{R}^{N}$ is a monotone homeomorphism such that

(a) $\phi(x)=a(x) \phi_{p}(x), \forall x \in \mathbb{R}^{N}$, with $\phi_{p}$ the wellknow $p$-Laplacian operator and $\nabla \Phi=\phi$ with $\Phi: \mathbb{R}^{N} \longrightarrow \mathbb{R}$ of class $C^{1}$ on $\mathbb{R}^{N}$, strictly convex (i.e., $\Phi$ is a potential function corresponding to $\phi$ ) and such that $\Phi(0)=0$;

(b) there exist $c_{1}, c_{2}, c_{3}>0$ such that

$$
c_{1}|x|^{p} \leq \Phi(x) \leq c_{2}+c_{3}|x|^{p}, \quad \forall x \in \mathbb{R}^{N},
$$

where $|$.$| denotes the Euclidean norm on \mathbb{R}^{N}$.

Remark 1. Suppose that $\forall x \in \mathbb{R}^{N}, a(x)=1$, we have $\phi(x)=\phi_{p}(x)=|x|^{p-2} x, p>1$. Then this function satisfies hypotheses $\left(H_{a}\right)$ and $\left(H_{\phi}\right)$. Other cases that satisfy Hypotheses $\left(H_{a}\right)$ and $\left(H_{\phi}\right)$ are when $\eta<a(x)<M$ with $\eta, M>0$ and the function $\phi: x \longmapsto a(x)|x|^{p-2} x$ is a monotone homeomorphism on $\mathbb{R}^{N}$. This is the case, for example, when function $a$ is equal to one of the following functions:

$$
\begin{gathered}
x \longmapsto 1+\frac{1}{\left(1+|x|^{p}\right)^{2}} \\
\text { or } x \longmapsto p+\frac{p-|x|}{e^{|x|}}, \quad \forall x \in \mathbb{R}^{N},
\end{gathered}
$$

with $p>1$. Indeed, for these examples, the potentials functions $\Phi$ corresponding to $\phi$ are respectively the functions $x \longmapsto(1 / p)\left(1+\left(1 /\left(1+|x|^{p}\right)\right)\right)|x|^{p}$ and $x \longmapsto(1+$ $\left.\left(1 / e^{|x|}\right)\right)|x|^{p}, \forall x \in \mathbb{R}^{N}$.

$\left(H_{F}\right): \quad F:(0, T) \times \mathbb{R}^{N} \longrightarrow \mathbb{R}^{N}$ is a Caratheodory mapping such that

$\left(H_{F}\right)_{1}: F(t,$.$) is continuously differentiable, for a.e.$ $t \in(0, T)$

$\left(H_{F}\right)_{2}: F(., 0) \in L^{1}(0, T)$

$\left(H_{F}\right)_{3}$ : for each $R>0$ there is some $\alpha_{R} \in L^{1}(0, T)$ such that

$$
|\nabla F(t, x)| \leq \alpha_{R}(t), \quad \text { a.e. } t \in(0, T), \forall x \in \mathbb{R}^{N} \text { with }|x| \leq R \text {. }
$$

Our notion of solution of problem (1) is defined as follows:

Definition 1. By a solution of the differential system (1), we will understand a function $u:[0, T] \longrightarrow \mathbb{R}^{N}$ of class 
$C^{1}\left(\Omega, \mathbb{R}^{N}\right)$ with $\phi\left(u^{\prime}\right)$ absolutely continuous, which satisfies the equality in (1) a.e. on $(0, T)$.

Now let us start the variational approach. In this purpose, for $\varepsilon>0$, let $K_{\varepsilon}: W^{1, p}\left((0, T), \mathbb{R}^{N}\right) \longrightarrow \mathbb{R}$ be defined by

$$
K_{\varepsilon}(u)=\int_{0}^{T}\left(\Phi\left(u^{\prime}(s)\right)+\varepsilon \Phi(u(s))\right) \mathrm{d} s, \quad \forall u \in W^{1, p}\left((0, T), \mathbb{R}^{N}\right) .
$$

Lemma 1. $K_{\varepsilon}$ is proper, convex, and lower semicontinuous.

Proof. By hypothesis $\left(H_{\phi}\right)$, it follows

$$
\begin{array}{r}
c_{1}\left(\left\|u^{\prime}\right\|_{p}^{p}+\varepsilon\|u\|_{p}^{p}\right) \leq K_{\varepsilon}(u) \leq(1+\varepsilon) c_{2}+c_{3}\left(\left\|u^{\prime}\right\|_{p}^{p}+\varepsilon\|u\|_{p}^{p}\right), \\
\forall u \in W^{1, p}\left((0, T), \mathbb{R}^{N}\right) .
\end{array}
$$

Whence $K_{\varepsilon}$ is proper. Also since $\Phi$ is convex, it follows that $K_{\varepsilon}$ is convex. Finally, because of the lower semicontinuity of the functional norm on Banach space, $K_{\varepsilon}$ is lower semicontinuous (in short, lsc).

Lemma 2. $K_{\varepsilon} \in C^{1}\left(W^{1, p}\left((0, T), \mathbb{R}^{N}\right), \mathbb{R}\right)$ and

$$
\begin{array}{r}
\left\langle K_{\varepsilon}^{\prime}(u), w\right\rangle=\int_{0}^{T}\left\langle\phi\left(u^{\prime}(t)\right), w^{\prime}(t)\right\rangle_{\mathbb{R}^{N}}+\varepsilon\langle\phi(u(t)), w(t)\rangle_{\mathbb{R}^{N}} \mathrm{~d} t, \\
\forall u, w \in W^{1, p}\left((0, T), \mathbb{R}^{N}\right),
\end{array}
$$

where \langle\rangle$_{\mathbb{R}^{N}}$ is the inner product in $\mathbb{R}^{N}$.

Proof. Let us consider the product space $H=\prod_{i=1}^{N} L^{q}(\Omega, \mathbb{R})$, $(1 / p)+(1 / q)=1$, equipped with the norm

$$
\|x\|_{H}=\left(\sum_{i=1}^{N}\left\|x_{i}\right\|_{q}^{q}\right)^{1 / q}, \quad \forall x=\left(x_{1}, x_{2}, \ldots, x_{n-1}, x_{n}\right) \in H .
$$

We define $g\left(g_{1}, g_{2}, \ldots, g_{n-1}, g_{N}\right): W^{1, p}\left((0, T), \mathbb{R}^{N}\right)$ $\longrightarrow H$ such that

$$
g(u)=\phi\left(u^{\prime}\right)+\varepsilon \phi(u), \quad \forall u \in W^{1, p}\left((0, T), \mathbb{R}^{N}\right) .
$$

Let us show that $g$ is bounded.

We have

$$
\begin{aligned}
\left\|g_{i}(u)\right\|_{q}^{q}= & \int_{0}^{T}\left|\phi_{i}\left(u^{\prime}(t)\right)+\varepsilon \phi_{i}(u(t))\right|^{q} \mathrm{~d} t \\
= & \left.\int_{0}^{T}\left|a\left(u^{\prime}(t)\right)\right| u^{\prime}(t)\right|^{p-2} u_{i}^{\prime}(t) \\
& +\left.\varepsilon a(u(t))|u(t)|^{p-2} u_{i}(t)\right|^{q} \mathrm{~d} t \\
\leq & M^{q} \int_{0}^{T}\left|u^{\prime}(t)\right|^{(p-1) q}+\varepsilon \mid\left(\left.u(t)\right|^{(p-1) q} \mathrm{~d} t\right. \\
= & M^{q}\left(\left\|u^{\prime}\right\|_{p}^{p}+\varepsilon\|u\|_{p}^{p}\right)=M^{q}\|u\|_{\varepsilon}^{p} \\
\leq & M^{q} \eta_{1}\|u\|_{m}^{p}, \quad \text { for some } \eta_{1}>0 .
\end{aligned}
$$

It follows

$$
\|g(u)\|_{H} \leq M\left(N \eta_{1}\right)^{1 / q}\|u\|_{m}^{p-1}, \quad \forall u \in W^{1, p}(\Omega) .
$$

So $g$ is bounded.

Let us show that $g$ is continuous.

Let $\left\{u_{n}\right\}_{n \geq 1}$ be a sequence such that $u_{n} \longrightarrow u$ in $W^{1, p}(\Omega)$. Then $u_{n} \longrightarrow u$ in $L^{p}(\Omega)$ and $u_{n}^{\prime} \rightarrow u^{\prime}$ in $L^{p}(\Omega)$. Whence $\phi_{i}\left(u_{n}^{\prime}\right) \longrightarrow \phi_{i}\left(u^{\prime}\right)$ in $L^{q}(\Omega)$ and $\phi_{i}\left(u_{n}\right) \longrightarrow \phi_{i}(u)$ in $L^{q}(\Omega)$. We set

$$
g_{i}\left(u_{n}\right)=\phi_{i}\left(u_{n}^{\prime}\right)+\varepsilon \phi_{i}\left(u_{n}\right) .
$$

By the previous arguments, the sequence $g_{i}\left(u_{n}\right) \longrightarrow$ $g_{i}(u)$ in $L^{q}(\Omega)$. We infer that the sequence $g\left(u_{n}\right) \longrightarrow g(u)$ in $H$. So $g$ is continuous.

We consider the functional:

$$
Q(v)=\int_{0}^{T}\left\langle\phi\left(u^{\prime}(t)\right), v^{\prime}(t)\right\rangle+\varepsilon\langle\phi(u(t)), v(t)\rangle \mathrm{d} t,
$$
for all $v \in W^{1, p}(\Omega)$.

Let us show that the linear operator $Q$ is continuous on $W^{1, p}(\Omega)$.

Using Hölder's inequality, we obtain

$$
\begin{aligned}
|Q(v)|= & \left|\int_{0}^{T}\left\langle\phi\left(u^{\prime}(t)\right), v^{\prime}(t)\right\rangle+\varepsilon\langle\phi(u(t)), v(t)\rangle \mathrm{d} t\right| \\
\leq & \left(\int_{0}^{T}\left|\phi\left(u^{\prime}(t)\right)\right|^{q} \mathrm{~d} t\right)^{1 / q}\left(\int_{0}^{T}\left|v^{\prime}(t)\right|^{p} \mathrm{~d} t\right)^{1 / p} \\
& +\varepsilon\left(\int_{0}^{T}|\phi(u(t))|^{q} \mathrm{~d} t\right)^{1 / q}\left(\int_{0}^{T}|v(t)|^{p} \mathrm{~d} t\right)^{1 / p} \\
\leq & M(1+\varepsilon)\|u\|_{m}^{p / q}\|v\|_{m}, \quad \forall u \in W^{1, p}(\Omega) .
\end{aligned}
$$


So $Q$ is continuous.

Let us show that $K_{\varepsilon}$ is Frechet differentiable in $u \in W^{1, p}(\Omega)$ and $K_{\varepsilon}^{\prime}(u)=\phi\left(u^{\prime}\right)+\varepsilon \phi(u)$ in the sense of (15).
Using Fubini's inequality, for arbitrary $v \in W^{1, p}(\Omega)$, we obtain

$$
\begin{aligned}
K_{\varepsilon}( & +v)-K_{\varepsilon}(u)-\int_{0}^{T}\left\langle\phi\left(u^{\prime}(t)\right), v^{\prime}(t)\right\rangle+\varepsilon\langle\phi(u(t)), v(t)\rangle \mathrm{d} t \\
= & \int_{0}^{T} \int_{0}^{1} \frac{\mathrm{d}}{\mathrm{d} s}\left(\Phi\left(u^{\prime}(t)+s v^{\prime}(t)\right)\right)+\varepsilon \frac{\mathrm{d}}{\mathrm{d} s}(\Phi(u(t)+s v(t))) \mathrm{d} s \mathrm{~d} t \\
& -\int_{0}^{T} \int_{0}^{1}\left\langle\phi\left(u^{\prime}(t)\right), v^{\prime}(t)\right\rangle+\varepsilon\langle\phi(u(t)), v(t)\rangle \mathrm{d} s \mathrm{~d} t \\
= & \int_{0}^{T} \int_{0}^{1}\left\langle\phi\left(u^{\prime}(t)+s v^{\prime}(t)\right), v^{\prime}(t)\right\rangle+\varepsilon\langle\phi(u(t)+s v(t)), v(t)\rangle \mathrm{d} s \mathrm{~d} t \\
& -\int_{0}^{T} \int_{0}^{1}\left\langle\phi\left(u^{\prime}(t)\right), v^{\prime}(t)\right\rangle+\varepsilon\langle\phi(u(t)), v(t)\rangle \mathrm{d} s \mathrm{~d} t \\
= & \int_{0}^{T} \int_{0}^{1}\left\langle\phi\left(u^{\prime}(t)+s v^{\prime}(t)\right)-\phi\left(u^{\prime}(t)\right), v^{\prime}(t)\right\rangle+\varepsilon\langle\phi(u(t)+s v(t))-\phi(u(t)), v(t)\rangle \mathrm{d} s \mathrm{~d} t \\
\leq & \int_{0}^{1}\left[\left(\int_{0}^{T}\left|\phi\left(u^{\prime}(t)+s v^{\prime}(t)\right)-\phi\left(u^{\prime}(t)\right)\right|^{q}\right)^{1 / q} \mathrm{~d} t\left(\int_{0}^{T}\left|v^{\prime}(t)\right|^{p}\right)^{1 / p} \mathrm{~d} t\right] \mathrm{d} s \\
\leq & \int_{0}^{1}\left(\left\|\phi\left(u^{\prime}+s v^{\prime}\right)-\phi\left(u^{\prime}\right)\right\|_{H}+\varepsilon\|\phi(u+s v)-\phi(u)\|_{H}\right) \mathrm{d} s\|v\|_{m} \\
& +\varepsilon \int_{0}^{1}\left[\left(\int_{0}^{T}|\phi(u(t)+s v(t))-\phi(u(t))|^{q}\right)^{1 / p} \mathrm{~d} t\left(\int_{0}^{T}|v(t)|^{p}\right)^{1 / p} \mathrm{~d} t\right] \mathrm{d} s
\end{aligned}
$$

with $L: W^{1, p}(\Omega) \longrightarrow H \quad$ and $\quad L(u)=\phi\left(u^{\prime}\right)$; $\Lambda: W^{1, p}(\Omega) \longrightarrow H$ and $\Lambda(u)=\phi(u)$.

continuous and bounded on $W^{1, p}(\Omega)$. Moreover, using

Arguing as in the proof of the continuity of $g$ and the fact it is bounded on $W^{1, p}(\Omega)$, we show that $L$ and $\Lambda$ are

$$
\begin{aligned}
& \frac{K_{\varepsilon}(u+v)-K_{\varepsilon}(u)-\int_{0}^{T}\left\langle\phi\left(u^{\prime}(t)\right), v^{\prime}(t)\right\rangle+\varepsilon\langle\phi(u(t)), v(t)\rangle \mathrm{d} t}{\|v\|_{m}} \\
& \quad \leq \int_{0}^{1}\left(\|L(u+s v)-L(u)\|_{H}+\varepsilon\|\Lambda(u+s v)-\Lambda(u)\|_{H}\right) \mathrm{d} s \longrightarrow 0, \quad \text { as }\|v\|_{m} \longrightarrow 0 .
\end{aligned}
$$

Therefore, (15) is proved.

We know that $K_{\varepsilon}^{\prime}: W^{1, p}(\Omega) \longrightarrow\left(W^{1, p}(\Omega)\right)^{*}$ where $\left(W^{1, p}(\Omega)\right)^{*}$ denotes the dual of $W^{1, p}(\Omega)$.

Let us show that $K_{\varepsilon}^{\prime}$ is continuous.

We have

$$
\begin{aligned}
\left\langle K_{\varepsilon}^{\prime}(u)-K_{\varepsilon}^{\prime}(v), w\right\rangle= & \int_{0}^{T}\left\langle\phi\left(u^{\prime}(t)\right)+\varepsilon \phi(u(t))\right. \\
& \left.-\phi\left(v^{\prime}(t)\right)-\varepsilon \phi(v(t)), w\right\rangle \mathrm{d} t \\
= & \int_{0}^{T}\langle g(u)(t)-g(v)(t), w\rangle \mathrm{d} t .
\end{aligned}
$$


Using Hölder's inequality, we have

$$
\begin{array}{r}
\left\langle K_{\varepsilon}^{\prime}(u)-K_{\varepsilon}^{\prime}(v), w\right\rangle \leq k\|g(u)-g(v)\|_{H}\|w\|_{m}, \\
\forall u, v, w \in W^{1, p}(\Omega) .
\end{array}
$$

Whence

$$
\left\|K_{\varepsilon}^{\prime}(u)-K_{\varepsilon}^{\prime}(v)\right\|_{\left(W^{1, p}(\Omega)\right)^{*}} \leq k\|g(u)-g(v)\|_{H} .
$$
$K_{\varepsilon}^{\prime}$

Thus, since $g$ is continuous, we obtain the continuity of We introduce also the functional: $J: W^{1, p}((0, T)$, $\left.\mathbb{R}^{N}\right) \longrightarrow(-\infty,+\infty]$ defined by

$$
J(u)=j(u(0), u(T)), \quad \forall u \in W^{1, p}\left((0, T), \mathbb{R}^{N}\right) .
$$

Recall that $j$ is proper, convex, and lsc. Then, $J$ is also proper, convex, and lsc. Let us set

$$
\Delta_{\varepsilon}=K_{\varepsilon}+J .
$$

Since $K_{\varepsilon}$ and $J$ are proper, convex and lsc, it follows that $\Delta_{\varepsilon}$ is proper, convex and lsc on $W^{1, p}\left((0, T), \mathbb{R}^{N}\right)$. Assuming that hypotheses $\left(H_{F}\right)_{1},\left(H_{F}\right)_{2}$ and $\left(H_{F}\right)_{3}$ on the Caratheodory function $F$ hold, for each $R>0$, we obtain:

$|F(t, x)| \leq R \alpha_{R}(t)+|F(t, 0)|, \quad$ for a.e $t \in(0, T)$ with $|x| \leq R$,

with $\alpha_{R} \in L^{1}(0, T)$. Equation (30) comes from inequality (12) and the estimation:

$$
\begin{aligned}
|F(t, x)| & =\left|\int_{0}^{1} \frac{\mathrm{d}}{\mathrm{d} s} F(t, s x) \mathrm{d} s\right|+F(t, 0) \\
& \leq\left|\int_{0}^{1}\langle\nabla F(t, s x), x\rangle_{\mathbb{R}^{N}} \mathrm{~d} s\right|+|F(t, 0)| \\
& \leq|x|\left|\int_{0}^{1} \nabla F(t, s x) \mathrm{d} s\right|+|F(t, 0)|,
\end{aligned}
$$

equation (30) and the embedding $W^{1, p}\left((0, T), \mathbb{R}^{N}\right) \subset$ $C\left((0, T), \mathbb{R}^{N}\right)$ allow us to introduce the functional: $\Psi_{F}: W^{1, p}\left((0, T), \mathbb{R}^{N}\right) \longrightarrow \mathbb{R}$ defined by

$$
\begin{aligned}
& \Psi_{F}(u)=-\int_{0}^{T} F(t, u(t)) \mathrm{d} t+\int_{0}^{T} F(t, 0) \mathrm{d} t, \\
& \forall u \in W^{1, p}\left((0, T), \mathbb{R}^{N}\right) .
\end{aligned}
$$

Lemma 3. If the hypotheses $\left(H_{F}\right)_{1},\left(H_{F}\right)_{2}$, and $\left(H_{F}\right)_{3}$ hold, then

$$
\begin{aligned}
\Psi_{F} \in C^{1}\left(W^{1, p}\left(\Omega, \mathbb{R}^{N}\right), \mathbb{R}\right), \\
\left\langle\Psi_{F}^{\prime}(u), v\right\rangle=-\int_{0}^{T}(\nabla F(t, u), v) \mathrm{d} t, \\
\forall u, v \in W^{1, p}\left((0, T), \mathbb{R}^{N}\right) .
\end{aligned}
$$

Proof. The proof is similar to the one of Theorem 2.6 of [2].
Considering the functional framework in Section 2, we set $X=W^{1, p}\left((0, T), \mathbb{R}^{N}\right), E=\Psi_{F}$ in (32), $G=\Delta_{\varepsilon}$ in (29) and $B_{F, \varepsilon}=B$ :

$$
B_{F, \varepsilon}=\Psi_{F}+\Delta_{\varepsilon}
$$

Proposition 2. Let the Caratheodory function $F:(0, T) \times$ $\mathbb{R}^{N} \longrightarrow \mathbb{R}$ satisfies $\left(H_{F}\right)_{1},\left(H_{F}\right)_{2}$, and $\left(H_{F}\right)_{3}$ and let $u \in W^{1, p}\left((0, T), \mathbb{R}^{N}\right)$. If $u$ is a critical point of the functional $B_{F, \varepsilon}$ defined by (35), in the sense (7), i.e.,

$$
\begin{array}{r}
\left\langle\Psi_{F}^{\prime}(u), w-u\right\rangle+\Delta_{\varepsilon}(w)-\Delta_{\varepsilon}(u) \geq 0, \\
\forall w \in W^{1, p}\left((0, T), \mathbb{R}^{N}\right),
\end{array}
$$

then $u$ is a solution of problem (1). The converse implication is also true.

Proof. We suppose that $u$ is a critical point of $B_{F, \varepsilon}$. In the inequality (36), we take $w=u+s v$. Then dividing by $s$ and letting $s \longrightarrow 0^{+}$, we obtain

$$
\begin{array}{r}
\left\langle\Psi_{F}^{\prime}(u), v\right\rangle+\left\langle K_{\varepsilon}^{\prime}(u), v\right\rangle+J^{\prime}(u ; v) \geq 0, \\
\forall v \in W^{1, p}\left((0, T), \mathbb{R}^{N}\right),
\end{array}
$$

where $J^{\prime}(u ; v)$ is the directional derivative of the convex function $J$ at $u$ in the direction $v$. From (28) and (37), it follows

$$
\begin{aligned}
& \left\langle\Psi_{F}^{\prime}(u), v\right\rangle+\left\langle K_{\varepsilon}^{\prime}(u), v\right\rangle+j^{\prime}((u(0), u(T)) ; \\
& \quad(v(0), v(T))) \geq 0, \quad \forall v \in W^{1, p}\left((0, T), \mathbb{R}^{N}\right) .
\end{aligned}
$$

Since $\quad C_{0}^{\infty}\left((0, T), \mathbb{R}^{N}\right) \subset W^{1, p}\left((0, T), \mathbb{R}^{N}\right), \quad$ using Hahn-Banach's theorem (see Theorem I.1 of Brezis [3]), (38) implies

$$
\left\langle\Psi_{F}^{\prime}(u), v\right\rangle+\left\langle K_{\varepsilon}^{\prime}(u), v\right\rangle=0, \quad \forall v \in C_{0}^{\infty}\left((0, T), \mathbb{R}^{N}\right) .
$$

Using (15), (34), and (39), we obtain

$$
\begin{array}{r}
\int_{0}^{T}\left\langle\phi\left(u^{\prime}(t)\right), v^{\prime}(t)\right\rangle_{\mathbb{R}^{N}} \mathrm{~d} t \\
=\int_{0}^{T}\langle-\varepsilon \phi(u(t))+\nabla F(t, u(t)), v(t)\rangle_{\mathbb{R}^{N}} \mathrm{~d} t, \\
\forall v \in C_{0}^{\infty}\left((0, T), \mathbb{R}^{N}\right) .
\end{array}
$$

From hypothesis $\left(H_{\phi}\right)(b)$ and $u \in W^{1, p}\left((0, T), \mathbb{R}^{N}\right)$, it follows that

$$
\phi(u), \phi\left(u^{\prime}\right) \in L^{q}\left((0, T), \mathbb{R}^{N}\right), \quad \text { with } \frac{1}{p}+\frac{1}{q}=1 .
$$

Also, $\left(H_{F}\right)_{3}$ implies

$$
\nabla F(., u) \in L^{1}\left((0, T), \mathbb{R}^{N}\right) .
$$

Equations (40) and (42) imply

$$
\begin{gathered}
\phi\left(u^{\prime}\right) \in W^{1,1}\left((0, T), \mathbb{R}^{N}\right), \\
-\left(\phi\left(u^{\prime}(t)\right)\right)^{\prime}=-\varepsilon \phi(u(t))+\nabla F(t, u(t)) .
\end{gathered}
$$


With $\phi$ being a homeomorphism, (43) ensures that $u \in C^{1}\left((0, T), \mathbb{R}^{N}\right)$. This together with (44) shows that $u$ is the solution of the differential system (1). Furthermore, (38) and (44) yield

$$
\begin{aligned}
j^{\prime}((u(0), u(T)) ;(v(0), v(T))) \geq & \left\langle\phi\left(u^{\prime}(0)\right), v(0)\right\rangle \\
& -\left\langle\phi\left(u^{\prime}(T)\right), v(T)\right\rangle, \\
& \forall v \in W^{1, p}\left((0, T), \mathbb{R}^{N}\right) .
\end{aligned}
$$

Thus,

$$
\begin{gathered}
j^{\prime}((u(0), u(T)) ;(x, y)) \geq\left\langle\phi\left(u^{\prime}(0)\right), x\right\rangle-\left\langle\phi\left(u^{\prime}(T)\right), y\right\rangle, \\
\forall x, y \in \mathbb{R}^{N},
\end{gathered}
$$

which, by a standard result from convex analysis (Theorem 23.2 of Rockafellar [8]), means that the boundary conditions in (1) are true.

Now let us show the converse implication.

By multiplying (1) with $v \in W^{1, p}\left((0, T), \mathbb{R}^{N}\right)$ and integrating by parts on $(0, T)$, we obtain

$$
\begin{aligned}
& \int_{0}^{T}\left(\left\langle\phi\left(u^{\prime}(t)\right), v^{\prime}(t)\right\rangle_{\mathbb{R}^{N}}+\varepsilon\langle\phi(u(t)), v(t)\rangle_{\mathbb{R}^{N}}\right) \mathrm{d} t \\
& \quad+\left\langle\phi\left(u^{\prime}(0)\right), v^{\prime}(0)\right\rangle_{\mathbb{R}^{N}}-\left\langle\phi\left(u^{\prime}(T)\right), v^{\prime}(T)\right\rangle_{\mathbb{R}^{N}} \\
& =\int_{0}^{T}\langle\nabla F(t, u(t)), v(t)\rangle_{\mathbb{R}^{N}} \mathrm{~d} t .
\end{aligned}
$$

Using inequalities (15) and (34) in (47), it follows

$$
\begin{aligned}
& \left\langle\Psi_{F}^{\prime}(u), v\right\rangle+\left\langle K_{\varepsilon}^{\prime}(u), v\right\rangle+\left\langle\phi\left(u^{\prime}(0)\right), v^{\prime}(0)\right\rangle_{\mathbb{R}^{N}} \\
& \quad-\left\langle\phi\left(u^{\prime}(T)\right), v^{\prime}(T)\right\rangle_{\mathbb{R}^{N}}=0 .
\end{aligned}
$$

By using Theorem 23.2 of Rockafellar [8], we obtain $\left\langle\Psi_{F}^{\prime}(u), v\right\rangle+\left\langle K_{\varepsilon}^{\prime}(u), v\right\rangle+j^{\prime}((u(0), u(T)) ;(v(0), v(T))) \geq 0$.

Using some previous arguments, we infer that

$$
\left\langle\Psi_{F}^{\prime}(u), w-u\right\rangle+\Delta_{\varepsilon}(w)-\Delta_{\varepsilon}(u) \geq 0, \quad \forall u \in W^{1, p}\left((0, T), \mathbb{R}^{N}\right) .
$$

\section{Existence Results for Problem (1)}

At first, let us introduce the constant

$$
\begin{aligned}
\lambda_{1}= & \lambda_{1}(p, j, \varepsilon)=\varepsilon \\
& +\inf \left\{\frac{\left\|u^{\prime}\right\|_{p}^{p}}{\|u\|_{p}^{p}}: u \in W^{1, p}\left((0, T), \mathbb{R}^{N}\right) \backslash\{0\},\right. \\
& (u(0), u(T)) \in D(j)\},
\end{aligned}
$$

for $\varepsilon \geq 0$. If $D(j)=\mathbb{R}^{N} \times \mathbb{R}^{N}$, then $\lambda_{1}(p, j, 0)=0$ and if $D(j)=\{(0 ; 0)\}$, then $\lambda_{1}(p, j, 0)>0$. To obtain the existence result, we make the following hypothesis:

$$
\left(H_{\lambda_{1}}\right) \lambda_{1}(p, j, \varepsilon)>0 \text {. }
$$

Proposition 3. If $\left(H_{\lambda_{1}}\right)$ is true, then

$$
2^{1 / p}\|u\|_{\lambda_{1}} \leq\left(\left\|u^{\prime}\right\|_{p}^{p}+\varepsilon\|u\|_{p}^{p}\right)^{1 / p} \leq\|u\|_{\lambda_{1}}, \quad \forall u \in D(J)
$$

where $J$ is defined by (28).

Proof. Since

$D(J)=\left\{u \in W^{1, p}\left((0, T), \mathbb{R}^{N}\right):(u(0), u(T)) \in D(j)\right\}, \quad$ by (51) we have

$$
\begin{aligned}
\lambda_{1}= & \lambda_{1}(p, j, \varepsilon)=\varepsilon+\inf \left\{\frac{\left\|u^{\prime}\right\|_{p}^{p}}{\|u\|_{p}^{p}}: u \in W^{1, p}\left((0, T), \mathbb{R}^{N}\right) \backslash\{0\},\right. \\
& (u(0), u(T)) \in D(j)\} .
\end{aligned}
$$

Whence,

$$
\begin{array}{r}
\lambda_{1} \leq \varepsilon+\frac{\left\|u^{\prime}\right\|_{p}^{p}}{\|u\|_{p}^{p}} \forall u \in W^{1, p}\left((0, T), \mathbb{R}^{N}\right) \backslash\{0\}, \\
(u(0), u(T)) \in D(j) .
\end{array}
$$

Then

$$
\lambda_{1}\|u\|_{p}^{p} \leq \varepsilon\|u\|_{p}^{p}+\left\|u^{\prime}\right\|_{p}^{p}
$$

It follows

$$
\left\|u^{\prime}\right\|_{p}^{p}+\lambda_{1}\|u\|_{p}^{p} \leq 2\left\|u^{\prime}\right\|_{p}^{p}+\varepsilon\|u\|_{p}^{p} .
$$

Whence

$$
2^{-(1 / P)}\|u\|_{\lambda_{1}} \leq\left(\left\|u^{\prime}\right\|_{p}^{p}+\varepsilon\|u\|_{p}^{p}\right)^{1 / p} .
$$

Furthermore, we have

$$
\varepsilon+\frac{\left\|u^{\prime}\right\|_{p}^{p}}{\|u\|_{p}^{p}} \leq \lambda_{1}+\frac{\left\|u^{\prime}\right\|_{p}^{p}}{\|u\|_{p}^{p}} .
$$

Then

$$
\left\|u^{\prime}\right\|_{p}^{p}+\varepsilon\|u\|_{p}^{p} \leq\left\|u^{\prime}\right\|_{p}^{p}+\lambda_{1}\|u\|_{p}^{p}
$$

So

$$
\left(\left\|u^{\prime}\right\|_{p}^{p}+\varepsilon\|u\|_{p}^{p}\right)^{1 / p} \leq\|u\|_{\lambda_{1}} .
$$

Inequalities (57) and (60) yield the result. 
If the nonlinearity $F$ lies asymptotically on the left of $\lambda_{1}$, then problem (1) is solvable.

Theorem 1. Assume $\left(H_{F}\right)_{1},\left(H_{F}\right)_{2},\left(H_{F}\right)_{3}$, and $H_{\lambda_{1}}$. If $\lim _{|x| \longrightarrow \infty} \sup \frac{F(t, x)}{c_{1}|x|^{p}}<\lambda_{1}, \quad$ uniformly for a.e. $t \in(0, T)$,

the problem (1) has at least a solution.

Proof. Let us show that the functional $B_{F, \varepsilon}$ in (35) is sequentially weakly lsc and coercive on the space $\left(W^{1, p}\left((0, T), \mathbb{R}^{N}\right),\|\|_{\lambda_{1}}\right)$.

Let us show that $\Psi_{F}$ in (32) is sequentially weakly continuous.

Let $u, v \in W^{1, p}\left((0, T), \mathbb{R}^{N}\right)$ be such that $\|u\|_{\infty},\|v\|_{\infty} \leq M$, with some $M>0$. By $\left(H_{F}\right)_{3}$, there is an $\alpha_{2 M} \in L^{1}(0, T)$ such that

$$
\begin{aligned}
|\nabla F(t, x)| & \leq \alpha_{2 M}, \\
& \text { for a.e } t \in(0, T), \forall x \in \mathbb{R}^{N} \text { with }\left|\alpha_{2 M}\right| \leq 2 M .
\end{aligned}
$$

We estimate

$$
\begin{aligned}
\left|\Psi_{F}(u)-\Psi_{F}(v)\right| & =\left|\int_{0}^{T}(F(t, u)-F(t, v))\right| \\
& =\left|\int_{0}^{T} \int_{0}^{1} \frac{\mathrm{d}}{\mathrm{d} s} F(t, u+s(v-u)) \mathrm{d} s\right| \\
& =\left|\int_{0}^{T} \int_{0}^{1}\langle\nabla F(t, u+s(v-u)), v-u\rangle_{\mathbb{R}^{N}} \mathrm{~d} s\right| \\
& \leq \int_{0}^{T} \int_{0}^{1}|\nabla F(t, u+s(v-u))| \mathrm{d} s\|u-v\|_{\infty},
\end{aligned}
$$

and by (62), it follows

$$
\left|\Psi_{F}(u)-\Psi_{F}(v)\right| \leq\left(\int_{0}^{T} \alpha_{2 M}(t) \mathrm{d} t\right)\|u-v\|_{\infty} .
$$

By the compactness of the embedding $W^{1, p}((0, T)$, $\left.\mathbb{R}^{N}\right) \subset C\left((0, T), \mathbb{R}^{N}\right)$ and (64), it follows that $\Psi_{F}$ is sequentially weakly continuous on $W^{1, p}\left((0, T), \mathbb{R}^{N}\right)$. Then, by the weak lower semicontinuity of $\Delta_{\varepsilon}$ in (29), $B_{F, \varepsilon}$ is sequentially weakly lower semicontinuous.

Furthermore, from (61), there are constant $\sigma \in\left(0, \lambda_{1}\right)$ and $\rho>0$ such that

$$
\begin{aligned}
F(t, x) \leq & \left(\lambda_{1}-\sigma\right) c_{1}|x|^{p}, \\
& \text { for a.e } t \in(0, T), \forall x \in \mathbb{R}^{N} \text { with }|x|>\rho .
\end{aligned}
$$

Then, (30) and (65) yield

$$
\begin{array}{r}
F(t, x) \leq \rho \alpha_{p}(t)+|F(t, 0)|+\left(\lambda_{1}-\sigma\right) c_{1}|x|^{p}, \\
\text { for a.e } t \in(0, T), \forall x \in \mathbb{R}^{N},
\end{array}
$$

which, by (32), give

$$
\Psi_{F}(u) \geq-\gamma(\rho)-\left(\lambda_{1}-\sigma\right) c_{1}\|u\|_{p}^{p}, \quad \forall u \in W^{1, p}\left((0, T), \mathbb{R}^{N}\right),
$$

with $\gamma(\rho)=\rho \int_{0}^{T} \alpha_{p}(t) \mathrm{d} t+2 \int_{0}^{T}|F(0, t)| \mathrm{d} t$. Using (29), (67), and (53) and the Proposition 3 , we estimate $B_{F, \varepsilon}$ on $D(J)$ as follows:

$$
\begin{aligned}
B_{F, \varepsilon}(u)= & \Psi_{F}(u)+\Delta_{\varepsilon}(u) \\
\geq & -\gamma(\rho)-\left(\lambda_{1}-\sigma\right) c_{1}\|u\|_{p}^{p}+c_{1}\left(\left\|u^{\prime}\right\|_{p}^{p}+\varepsilon\|u\|_{p}^{p}\right)+J(u) \\
\geq & -\gamma(\rho)-\left(\lambda_{1}-\sigma\right) c_{1} \frac{\left(\left\|u^{\prime}\right\|_{p}^{p}+\varepsilon\|u\|_{p}^{p}\right)}{\lambda_{1}} \\
& +c_{1}\left(\left\|u^{\prime}\right\|_{p}^{p}+\varepsilon\|u\|_{p}^{p}\right)+J(u) \\
= & -\gamma(\rho)+\frac{\sigma}{\lambda_{1}} c_{1}\left(\left\|u^{\prime}\right\|_{p}^{p}+\varepsilon\|u\|_{p}^{p}\right)+J(u) \\
\geq & -\gamma(\rho)+\frac{\sigma}{2 \lambda_{1}} c_{1}\|u\|_{\lambda_{1}}^{p}+J(u), \quad \forall u \in D(J) .
\end{aligned}
$$

Since $j$ is convex and lsc, it is bounded from below by an affine functional. Therefore, by (28) there are constants $k_{1}, k_{2}, k_{3} \geq 0$ such that

$$
\begin{array}{r}
B_{F, \varepsilon}(u) \geq-\gamma(\rho)+\frac{\sigma}{2 \lambda_{1}} c_{1}\|u\|_{\lambda_{1}}^{p}-k_{1}|u(0)|-k_{2}|u(T)|-k_{3}, \\
\forall u \in D(J) .
\end{array}
$$

From (69) and the continuity embedding of $W^{1, p}\left((0, T), \mathbb{R}^{N}\right) \subset C\left((0, T), \mathbb{R}^{N}\right)$, we obtain

$$
B_{F, \varepsilon}(u) \geq-\gamma(\rho)+\frac{\sigma}{2 \lambda_{1}} c_{1}\|u\|_{\lambda_{1}}^{p}-\bar{k}_{1}\|u\|_{\lambda_{1}}-\bar{k}_{2}\|u\|_{\lambda_{1}}-k_{3},
$$

$\forall u \in D(J)$.

with some $\bar{k}_{1}, \bar{k}_{2} \geq 0$. Consequently,

$$
B_{F, \varepsilon}(u) \longrightarrow+\infty, \quad \text { as }\|u\|_{\lambda_{1}} \longrightarrow \infty,
$$

meaning that $B_{F, \varepsilon}$ is coercive on $\left(W^{1, p},\|.\|_{\lambda_{1}}\right)$.

Since the functional $B_{F, \varepsilon}$ is sequentially weakly lsc and coercive, by a well-known result from calculus of variations, $B_{F, \varepsilon}$ is bounded from below and attains its infimum at some $u \in W^{1, p}\left((0, T), \mathbb{R}^{N}\right)$. Then, by Proposition 1 and $3, u$ is a solution of problem (1).

Remarque 2. The problem (1) incorporates classical problems of Dirichlet, Neumann, Periodic, antiperiodic, and Sturm-Louiville (see Remark 3.9 of Jebelean [5]). 


\section{Example}

We consider the following problem:

$$
\left\{\begin{array}{l}
-\left(\left(1+\frac{1}{\left(1+\left|u^{\prime}(t)\right|^{p}\right)^{2}}\right) \phi_{p}\left(u^{\prime}(t)\right)\right)^{\prime}+\varepsilon\left(1+\frac{1}{\left(1+\left|u^{\prime}(t)\right|^{p-1}\right)^{2}}\right) \phi_{p}(u(t))=\beta(t) \phi_{p}(u(t)), \\
\text { a.e on } \Omega=] 0, T[ \\
\left(\left(1+\frac{1}{\left(1+\left|u^{\prime}(0)\right|^{p}\right)^{2}}\right) \phi_{p}\left(u^{\prime}(0)\right),-\left(1+\frac{1}{\left(1+\left|u^{\prime}(T)\right|^{p}\right)^{2}}\right) \phi_{p}(u(T))\right) \in \partial j(u(0), u(T)),
\end{array}\right.
$$

where $\phi_{p}(x)=|x|^{p-2} x, \forall x \in \mathbb{R}^{N}$, is the well-known $p$-Laplacian operator. Let us set $\Phi(x)=(1 / p)(1+$ $\left.\left(1 /\left(1+|x|^{p}\right)\right)\right)|x|^{p}$. Whence $\nabla \Phi(x)=\phi(x)=(1+(1 /(1+$ $\left.\left.\left.|x|^{p}\right)^{2}\right)\right) \phi_{p}(x)$. When, $p>1$, we see that $\phi$ is a monotone homeomorphism such that $\phi(0)=0$. Moreover, $\Phi$ satisfies hypothesis $\left(H_{\phi}\right)(b)$. Therefore, by Theorem 1 , problem $(72)$ has at least a solution.

\section{Conclusion}

In this article, using variational method which relies on Szulkin theory, we have established existence for secondorder problems with multivalued boundary conditions. We give an example but more examples and applications can be given.

\section{Data Availability}

No data were used to support this study.

\section{Conflicts of Interest}

The authors declare no conflicts of interest regarding the publication of this paper.

\section{References}

[1] R. Bader and N. S. Papageorgioua, "Quasilinear vector differential equations with maximal monotone terms and nonlinear boundary conditions," Annales Polonici Mathematici, vol. 73, no. 1, pp. 69-92, 2000.

[2] D. A. Béhi, A. Adjé, and K. C. Goli, "Lower and upper solutions method for nonlinear second-order differential equations involving a $\phi$-Laplacian operator," African Diaspora Journal of Mathematics, vol. 22, no. 1, pp. 22-41, 2019.

[3] H. Brezis, Analyse Fonctionnelle: Théorie et Applications, Masson, Paris, France, 1983.

[4] L. Gasinski and N. S. Papageorgiou, "Nonlinear second-order multivalued boundary value problems," Proceedings Mathematical Sciences, vol. 113, no. 3, pp. 293-319, 2003.

[5] P. Jebelean, "Variational methods for ordinary $p$-Laplacian systems with potential boundary conditions," Advances in Differential Equations, vol. 13, no. 3-4, pp. 273-322, 2008.

[6] P. Jebelean and G. Morosanu, "Ordinary p-Laplacian systems with nonlinear boundary conditions," Journal of Mathematical Analysis and Applications, vol. 313, no. 2, pp. 738-753, 2006.
[7] A. Szulkin, "Minimax principles for lower semicontinuous functions and applications to nonlinear boundary value problems," Annales de l'Institut Henri Poincare (C) Non Linear Analysis, vol. 3, no. 2, pp. 77-109, 1986.

[8] R. T. Rockafellar, Convex Analysis, Princeton University Press, Princeton, NJ, USA, 1972. 


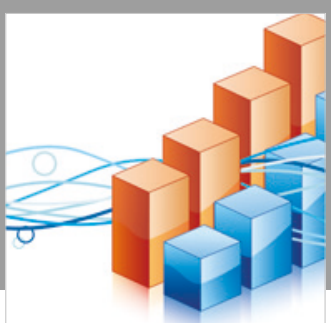

Advances in

Operations Research

\section{-n-m}
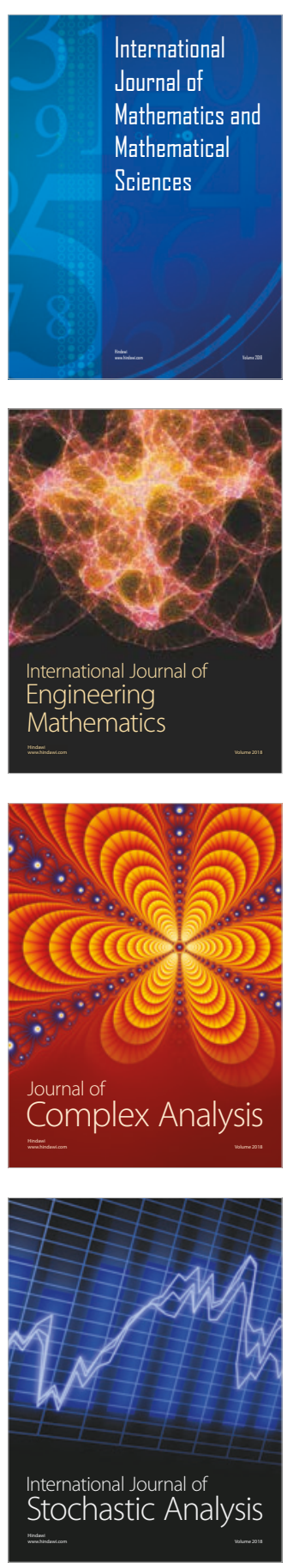
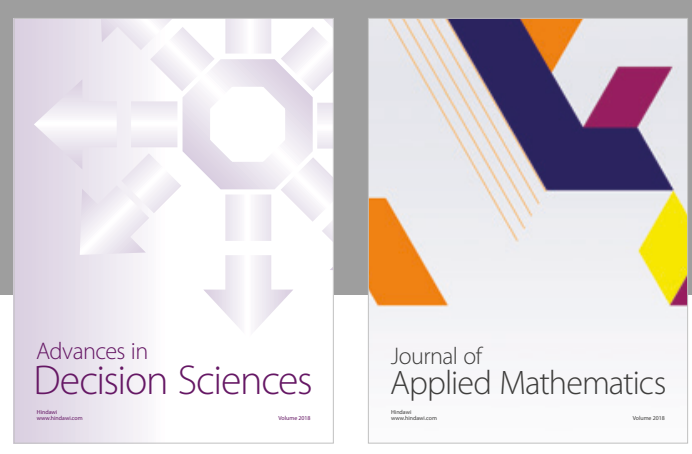

Journal of

Applied Mathematics
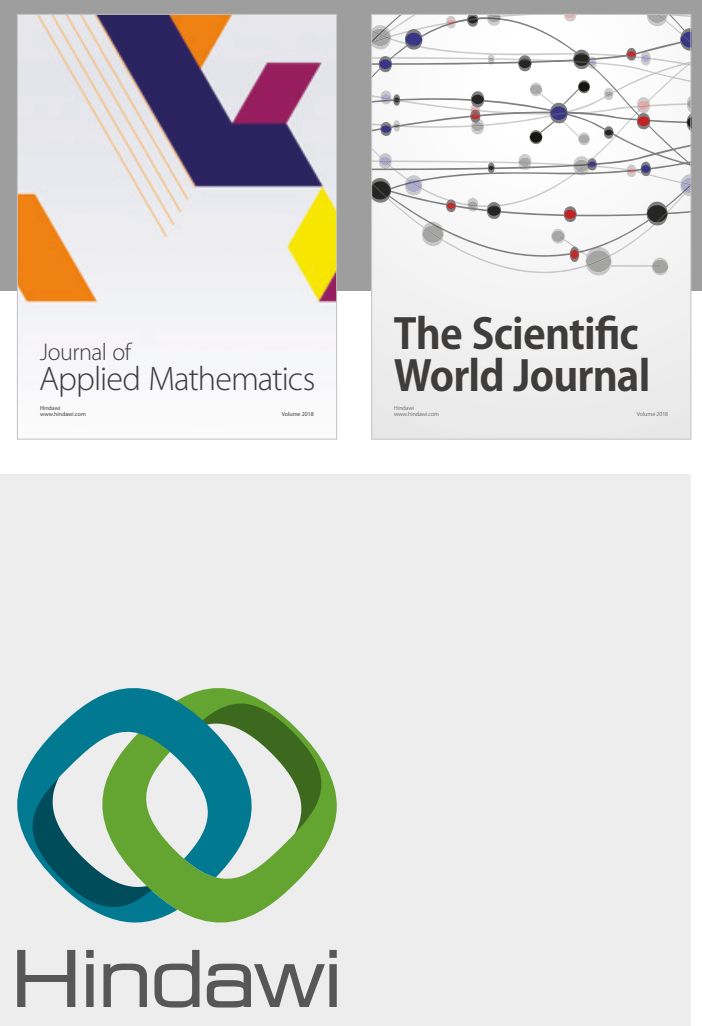

Submit your manuscripts at

www.hindawi.com

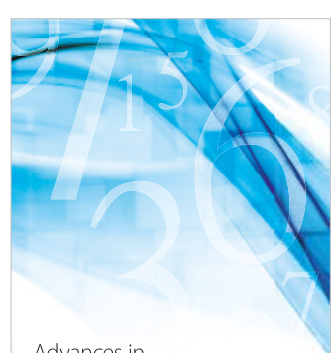

Advances in
Numerical Analysis
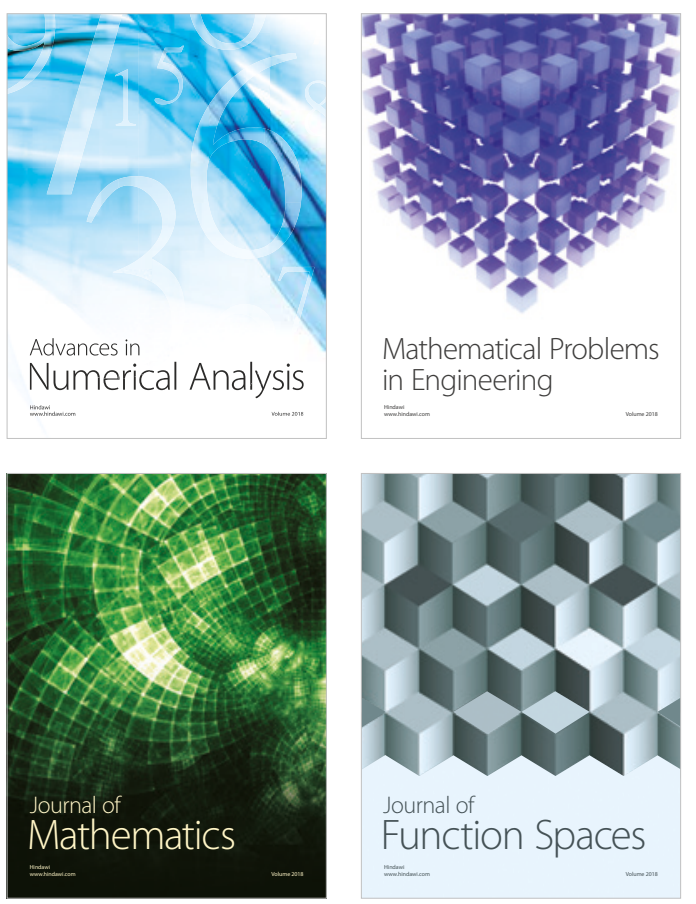

Mathematical Problems in Engineering

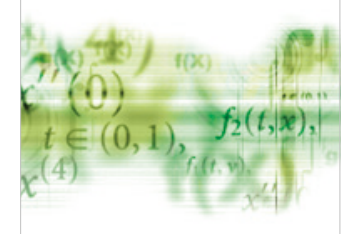

International Journal of

Differential Equations

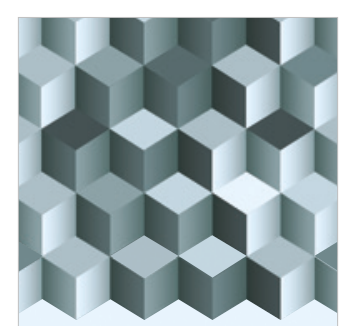

Journal of

Function Spaces

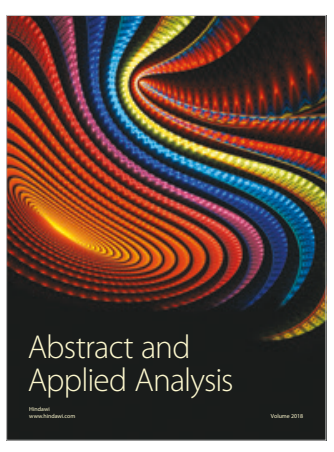

The Scientific

World Journal

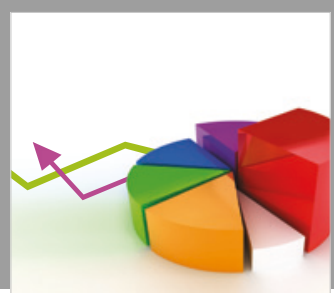

Journal of

Probability and Statistics
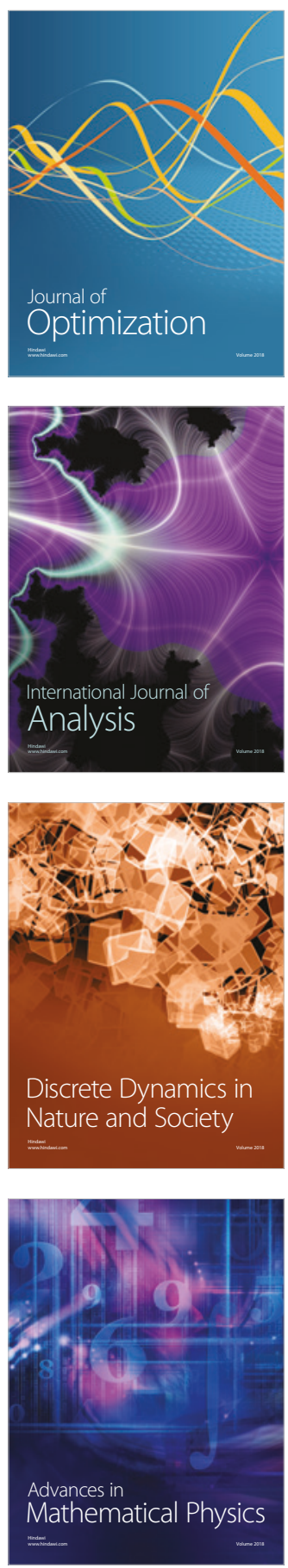